\title{
A Review on Antiphishing Framework
}

\author{
Sonal Akhare, Nikita Mayur, Vipashyna Kapse, Manjusha Harde, Deepak Kamde, Neha \\ Titarmare
}

Department of Computer Science, RGCER, Nagpur University, Maharashtra, India

\begin{abstract}
Phishing is an attack that deals with social engineering system to illegally get and utilize another person's information for the benefit of authentic site for possess advantage (e.g. Take of client's secret word and Visa precise elements during online correspondence). It is influencing all the significant areas of industry step by step with a considerable measure of abuse of client qualifications. To secure clients against phishing, different hostile to phishing procedures have been suggested that takes after various methodologies like customer side and server side insurance. In this paper we have considered phishing in detail (counting assault process and grouping of phishing assault) and investigated a portion of the current sites to phishing strategies alongside their points of interest and disadvantages.
\end{abstract}

Keywords - Anti-phishing, Authentication, Captcha, Image, legitimate.

\section{INTRODUCTION}

Online transactions are these days turn out to be extremely normal and there are different attacks exhibit behind this. In these sorts of different attacks, phishing is distinguished as a noteworthy security risk and new creative thoughts are emerging with this in every minute so preventive instruments ought to likewise be so usable. Therefore, the security in these cases be high and ought not to be effectively traceable with usage effortlessness. Phishing is an endeavour by an individual or a gathering to steal individual secret data, for example, Passwords and Credit card data and so forth. Programmers could make a clone of a site and instruct you to enter individual data, which is then messaged to them. Programmers generally exploit these locales to assault individuals utilizing them at their working environment, homes, or out in the open with a specific end goal to take individual and security data that can influence the client. Phishing tricks are additionally turning into an issue for internet managing an account and web based business clients. It is a criminal movement utilizing social Phishers endeavour to falsely secure delicate data, for example, passwords and charge card precise elements, by taking on the appearance of a reliable individual or business in an electronic correspondence. The harm brought on by phishing ranges from disavowal of access to email to significant money related misfortune. One of the essential objectives of phishing is to unlawfully do fake money related exchanges in the interest of clients utilizing a produced email that contains a URL indicating a fake site taking on the appearance of an online bank or an administration substance. A phisher may bait a casualty into giving his/her Social Security Number, full name, and address, which can then be utilized to apply for a charge card for the casualty's benefit. Assailant utilizes imitation of unique site as a snare that is send to the client. At the point when client snatches the goad by filling and presenting his helpful data assailant pulls lure implies spares the information for its own particular utilize wrongfully. All in all, phishing assaults are performed with the accompanying four stages:

1) A fake site which looks precisely like the honest to goodness Web webpage is set up by phisher.

2) Phisher then sends connection to the fake site in huge measure of parodied messages to target clients for the sake of true blue organizations and associations, attempting to persuade the potential casualties to visit their sites.

3) Victims visit the fake site by tapping on the connection and information its helpful data there.

4) Phisher then takes the individual data and play out their misrepresentation, for example, exchanging cash from the casualties' record.

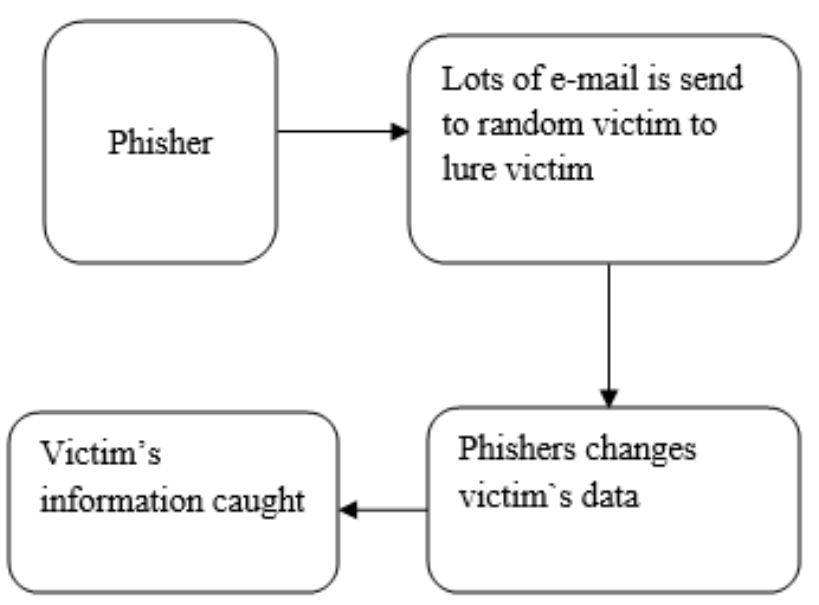

Fig.1: Process of Phishing 


\subsection{Types of Phishing}

\subsubsection{Email / Spam}

Phisher may send a similar email to a huge number of clients, asking for them to fill in individual subtle elements. These subtle elements will be utilized by the Phisher for their unlawful exercises. Phishing with email and spam is an exceptionally normal phishing trick. The vast majority of the messages have a critical note which requires the client to enter qualifications to upgrade account data, change subtle elements, and confirm accounts. Now and then, they might be made a request to round out a frame to get to another administration through a connection which is given in the email.

\subsubsection{Web Based Delivery}

Electronic conveyance is a standout amongst the most modern phishing strategies. Otherwise called "man-inthe-centre," the programme is situated in the middle of the first site and the phishing framework. The Phisher follows points of interest amid an exchange between the honest to goodness site and the client. As the client keeps on passing data, it is assembled by the Phisher, without the client thinking about it.

\subsubsection{Instant Messaging}

Instant Messaging is the strategy in which the client gets a message with a connection guiding them to a fake phishing site which has an indistinguishable look and feel from the true blue site. In the event that the client doesn't take a gander at the URL, it might be difficult to differentiate between the fake and true blue sites. At that point, the client is made a request to give individual data on the page.

\subsubsection{Trojan Hosts}

Trojan hosts are undetectable programmers attempting to sign into your client record to gather certifications through the neighbourhood machine. The obtained data is then transmitted to Phisher.

\subsubsection{Link Manipulation}

Link Manipulation is the method in which the Phisher sends a connection to a site. At the point when the client taps on the beguiling connection, it opens up the Phishers site rather than the site specified in the connection. One of the counter phishing systems used to anticipate interface control is to move the mouse over the connection to see the genuine address.

\subsubsection{Key Loggers}

Key loggers refer to the malware used to distinguish contributions from the console. The data is sent to the programmers who will interpret passwords and different sorts of data. To keep key lumberjacks from getting to individual data, secure sites give choices to utilize mouse snap to make passages through the virtual console.

\subsubsection{Session Hacking}

In session hacking, the Phisher exploits the web session control system to take data from the client. In a straightforward session hacking system known as session sniffing, the Phisher can utilize a sniffer to catch pertinent data so that he or she can get to the Web server unlawfully.

1.1.8 Phishing through Search Engines

Some phishing tricks include web search tools where the client is coordinated to items locales which may offer minimal effort items or administrations. At the point when the client tries to purchase the item by entering the visa subtle elements, it's gathered by the phishing site. There are many fake bank sites offering charge cards or advances to clients at a low rate however they are really phishing destinations.

\subsubsection{Phone Phishing}

In telephone phishing, the Phisher makes telephone calls to the client and requests that the client dial a number. The object is to get individual data of the financial balance through the telephone. Telephone phishing is generally finished with a fake guest ID.

\subsubsection{Malware Phishing}

Phishing tricks including malware oblige it to be keeping running on the client's PC. The malware is normally appended to the email sent to the client by the Phisher. When you tap on the connection, the malware will begin working. Infrequently, the malware may likewise be connected to downloadable records.

\subsubsection{Spear phishing}

Phishing endeavors coordinated at particular people or organizations have been named skewer assailants may accumulate individual data about their objective to build their likelihood of achievement. This procedure is, by a long shot, the best on the web today, representing $91 \%$ of assaults.

\subsubsection{Clone phishing}

Clone phishing is a kind of phishing assault whereby a genuine, and already conveyed, email containing a connection or connection has had its substance and beneficiary locations taken and used to make a practically indistinguishable or cloned email. The connection or connection inside the email is supplanted with a noxious form and afterward sent from an email deliver satirize to seem to originate from the first sender. It might claim to be a resend of the first or an upgraded form to the first. This method could be utilized to turn (by implication) from a formerly contaminated machine and pick up a decent footing on another machine, by misusing the social trust related with the derived association because of both sides getting the first email. 


\section{RELATED WORK}

2.1 Anti-phishing:

Anti-phishing refers to the strategy utilized so as to distinguish and counteract phishing attacks. Anti-phishing shields clients from phishing. A great deal of work has been done on against anti-phishing conceiving different opposed to phishing systems. A few systems deal with messages, a few takes a work at characteristics of locales and some on URL of the sites. A large number of these procedures concentrate on authorize customers to perceive and channel different sorts of phishing attacks. As a rule, opposed to phishing systems can be grouped into taking after four classifications.

2.1.1 Content Filtering - In this strategy content/email are filtered as it enters in the victim's mail box utilizing machine learning techniques, for example, Bayesian Additive Regression Trees (BART) or Support Vector Machines (SVM).

2.1.2 Black Listing - Blacklist is gathering of known phishing Web locales/addresses distributed by trusted substances like Google's and Microsoft's black list. It requires both a customer and a server part. The customer part is executed as either an email or program module that associates with a server segment, which for this situation is an open Web website that gives a rundown of known phishing webpage.

2.1.3 Symptom- Based Prevention - These prevention examinations the content of each Web page the client visits and produces phishing alerts indicated by the sort and number of symptoms recognized.

2.1.4 Domain Binding-It is a customer's program based strategies where touchy data (e.g. name, watchword) is tie to a specific area. It cautions the client when he visits an area to which client credential is not tie.

\subsection{Anti-phishing techniques}

\subsubsection{Attribute based anti-phishing techniques}

2.2.1.1 The Image Attribution check does an examination of images of visiting site and the sites officially enrolled with phish bouncer. The HTML Crosslink check from nonregistered destinations and connections the page has to any of the enrolled sites A high number of crossconnections is characteristic of a phishing site. In false data feeder check, false data is info and if that data is acknowledged by site then it is likely that connection is phished one.

2.2.1.2 The Certificate Suspicious check approves site testaments introduced during SSL handshake and broadens the average utilization by searching for Certification Authority (CA) consistency after some time. 2.2.1.3 URL suspicious check utilizes attributes of the Url to recognize phishing locales.

2.2.1.4 Advantage:
As characteristic based anti-phishing considers a ton of checks so it can identify more phished sites than different methodologies. It can distinguish referred to and also unknown attacks

2.2.1.5 Disadvantage:

As numerous checks perform to verify site this could bring about slow response time. Genetic Algorithm Based Anti-Phishing Techniques It is an approach of detection of phishing pages utilizing genetic algorithm.

2.2.2 Genetic algorithms:

Genetic algorithms can be utilized to evolve basic guidelines for preventing phishing attacks. These principles are utilized to separate typical site from atypical site. These odd sites suggest to occasions with probability of phishing attacks. The principles put away in the govern base are more often than not in the following structure.

2.2.2.1 Advantage:

It gives the component of malicious status warning before the client reads the mail. It additionally gives malicious web connect recognition what's more of phishing discovery.

2.2.2.2 Disadvantage:

Single rule for phishing recognition like in the case of url is a far way from enough, so we require various run set for just a single type of url based phishing discovery. In like manner, for other parameter we have to compose other administer this prompts to more complex calculation.

\subsubsection{An Identity Based Anti-Phishing Techniques}

This strategy takes after shared confirmation procedure where both client and online entities approves each other's identity during handshake. It is an anti-phishing method that coordinates halfway qualifications sharing and customer separating strategy to keep phishers from effortlessly taking on the appearance of authentic online element. As common verification is take process.

\subsubsection{Advantage:}

It gives mutual authentication for server and also customer side. Utilizing this systems client does not to reveal his credential password in entire session except from first time when the session is initialized.

\subsubsection{Disadvantage:}

In identity based anti-phishing if a programmer accesses the customer PC and disable the browser plug-in then technique will be compromise against phishing detection. 2.2.4 Character Based Anti-Phishing Approach

Many time phishers attempt to take data of clients by persuading them to tap on the hyperlink that they implant into phishing email. A hyperlink has a structure as takes after:

$<\mathrm{a}$ href $=$ "URI" > Anchor content $<\backslash \mathrm{a}>$ 
where "URI" (all inclusive asset identifiers) gives the real connection where the client will be coordinated and 'Grapple content' is the content that will be shown in client's Web program and speaks to the visual connection. Character based Antiphishing technique utilizes qualities of hyperlink keeping in mind the end goal to identify phishing joins. Connect protect is an apparatus that executes this system. In the wake of investigating numerous phishing sites, the hyperlinks can be arranged into different classifications. For discovery of phishing locales Link Guard, first concentrates the DNS names from the genuine and the visual connections and after that looks at the real and visual DNS names if these names are not the same, then it is phishing of classification 1 . On the off chance that spotted decimal IP address is specifically utilized as a part of real DNS, it is then a conceivable phishing assault of classification 2. On the off chance that the real connection or the visual connection is encoded (classes 3 and 4), then first the connection is decoded and after that broke down. At the point when there is no goal data (DNS name or spotted IP address) in the visual connection then the hyperlink is breaking down. Amid investigation DNS name is looked in boycott and white rundown. In the event that it is available in white list, then it is certain that the connection is bona fide and if connection is available in boycott then it is certain that connection is phished one. In the event that the real DNS is not contained in either white list or boycott, Pattern Matching is finished. Amid example coordinating first the sender email address is extricated and after that it is looked in seed set where a rundown of address is kept up that are physically gone to by the client. Similarly checks the most extreme probability of real DNS and the DNS names in seed-set. The similitude record between two strings is dictated by computing the negligible number of changes expected to change a string to the next string.

2.2.4.1 Advantage:

It can, distinguish referred to assaults, as well as is viable to the obscure ones. Tests demonstrated that Link Guard, can distinguish up to $96 \%$ obscure phishing assaults continuously to phishing assaults of classification 1 , it is certain that there is no false positives or false negatives. Connect Guard handles classifications 3 and 4 accurately since the encoded connections are initially decoded before further investigation.

\subsubsection{Disadvantage:}

For class 2, Link Guard may bring about false positives, since utilizing dabbed decimal IP addresses rather than area names might be alluring in some unique conditions.

\subsubsection{Content Based Anti-Phishing Approach}

Gold Phish instrument actualizes this procedure and utilizations Google as its internet searcher. This instrument gives higher rank to entrenched sites. It has been watched that phishing site pages are dynamic just for brief timeframe and along these lines will get low rank amid web scan and this gets to be reason for substance based hostile to phishing approach. The plan approach can be separated into three noteworthy strides. The initial step is to catch a picture of the present site in the client's web program. The second step is to utilize optical character acknowledgment procedures to change over the caught picture into PC discernable content. The third step is to enter the changed over content into a web crawler to recover comes about and break down the page rank.

2.2.5.1 Advantages:

By and large Gold Phish does not bring about false positive and gives zero-day phishing.

\subsubsection{Disadvantages:}

Gold Phish defers the rendering of a website page. It is additionally defenseless against assaults on Google's Page Rank calculation and Google's inquiry benefit.

\section{LITERATURE SURVEY}

The paper [1] was proposed by Divya James and Mintu Philip in the time of March 2015. In this paper we have learned about another approach named as "A Novel Antiphishing Framework Based on Visual Cryptography" to take care of the issue of phishing. Here an image based confirmation utilizing visual cryptography is investigated to protect the security of picture Captcha by deteriorating the first picture Captcha into two shares that are store in particular database server with the end goal that the first picture Captcha can be uncovered just when both are at the same time.

The paper [2] was displayed by prof. N R Jain, Kashid Ujwal, Shaikh Apsara, Patel Nikhil, Divekar Tejashri in year of April 2016.The OTP is get at a customer end just when the customer and dealer put their share. The OTP is checked by the bank server is distinguished Antiphishing, for identifying and counteracting phishing, they proposed another procedure for discovery of phishing site this will keep a client from being one of the casualty of the phishing assault. According to their strategy the dealer and the client ought to be enlisted to the bank.

The paper [3] was displayed by yanhui Du and Fu Xue in the year 2013.In this paper an Antiphishing method based on email extraction and examination is proposed. The procedure approach with phishing e-mail, the channel phishing assault transmits, distinguish phishing email and concentrate the suspicious URL from the email for further investigation. approach with phishing e-mail, the channel phishing assault transmit, phishing location framework gathers countless mail as the information source through a nectar pot insurance list without ensured site name is too in light of the fact that phishing can just actualize tricking 
by making utilization of this outsider which individuals are commonplace and trust.

Paper [4] was available by Ahmad Abbasi and Fatemeh "Mariam" Zahedi and Yan Chen in the yea June 2012.In this review, an examination including more than 400 participants were utilized to assess the effect of Antiphishing devices exactness on the client capacity to avoid phishing threats. Each of participants was given either a high accuracy or low accuracy tool and asked to make various decision about several legitimate and phishing websites, existing Antiphishing tools are fraud cause and blacklist to determine whether a particular website is legitimate or a phish fraud cause are website content, linkage and design elements that can serve as reliable indicator regarding the legitimacy of a website.

The paper [5] was presented by Srishti Gupt and Ponnurangam Kumara guru into the year of Jan 2014.each month more attack is launched with the aim of making web user believe that they are communicating with a trusted entity which compels them to share their personal financial information acquired sensitive information is then used for personal benefit's like gain access to money of the individual from whom the information was taken. phishing cost internet user billion, we found that Phishers have started to buy more number of domain, exploiting ICANN accredit registers and free sub domain registration service to launched attack we observed that phishing e - mail change over time with the Phishers using new techniques like propagation of promotional and money related $\mathrm{e}-$ mail to the people.

\section{PROPOSED WORK}

4.1 Registration Phase:

In enlistment stage the new client first must be enrolled with the framework, and enter the data of framework required. Here client need to choose picture as secret key and this picture will break into two impart one kept to client and another kept with the server.

4.2 Login Phase:

In login stage client need to enter their legitimate client name and secret key into the logging stage. At that point he needs to peruse the one share of picture and another from framework as a secret word. At the server side if both the picture of share match then it login into the record.

4.3 OTP (One Time Password) Generation:

In this stage if the client is validated client then OTP is sent to the client's enlisted portable number.

\section{CONCLUSION}

As of now phishing assaults are so basic since it can assault all inclusive and catch and stores the client's classified data. This data is utilized by the aggressors which are in a roundabout way required in the phishing process. Phishing site can be effectively recognized utilizing our proposed "Hostile to phishing system utilizing visual cryptography". This method gives extra security and limit outsider to get the private information. The proposed strategy is additionally helpful to keep the assaults of phishing site on monetary web portal, banking portal, online shopping market.

\section{REFERENCES}

[1] Divya James1 and Mintu Philip," A NOVEL ANTI PHISHING FRAMEWORK BASED ON VISUAL CRYPTOGRAPHY", IEEE transactions on Image Processing vol. 17 no, 2015, MTech in Information System Security, Indira Gandhi National Open University, India

[2] Prof N.R. Jain, Kashid Unwell, Shaikh Apsara, Patel Nikhil, Divekar Tejashri," ADVANCE PHISHING DETECTION USING VISUAL CRYPTOGRAPHY AND ONE TIME PASSWORD", International Journal of Advanced Research in Science, Engineering and Technology Vol. 3, Issue 4, April 2016, Department of Information \&Technology Engineering, PDEA'S College of Engineering Hadapsar, Pune, India.

[3] Yanhui Du," RESEARCH OF THE ANTIPHISHING TECHNOLOGY BASED ON E-MAIL EXTRACTION AND ANALYSIS", International Conference on Information Science and Cloud Computing Companion,2013

[4] Ahmed Abbasi; Fatemeh Mariam Zahedi and Yan chen" IMPACT OF ANTI-PHISHING TOOL PERFORMANCE ON ATTACK SUCCESS RATES”, ISI 2012, June 11-14, 2012, Washington, D.C., USA Information Technology University of Virginia Charlottesville, Virginia, USA.

[5] Srishti Gupta, Ponnurangam Kumara guru" EMERGING PHISHING TRENDS AND EFFECTIVENESS OF THE ANTI-PHISHING LANDING PAGE",2014, Indraprastha Institute of Information Technology, Delhi Cybersecurity Education and Research Centre (CERC), IIIT-Delhi 